\title{
PN-PAM scheme for short range optical transmission over SI-POF - an alternative to Discrete Multi-Tone (DMT) scheme
}

\author{
Linning Peng ${ }^{1}$, Ming Liu ${ }^{2 *}$, Maryline Hélard ${ }^{3}$ and Sylvain Haese ${ }^{3}$
}

\begin{abstract}
Background: How to deal with the time-dispersive channel is the main challenge faced by the short-range optical communication systems. In this work a novel pseudo-noise sequence (PN) assisted pulse-amplitude modulated (PN-PAM) transmission scheme for short-range optical communication systems is proposed in this work. With the help of the PN based channel estimation, minimum-phase pre-filter and reduced-state sequence estimation based equalizer, the proposed PAM transmission scheme can significantly reduce the training overhead for channel estimation in the classical PAM systems using decision feedback equalizer (DFE). In addition, the proposed PAM transmission scheme can effectively avoid the error propagation phenomenon in the classical DFE.

Results: Theoretical study shows that the proposed PAM scheme can achieve a 1.5 dB SNR gain with PAM-8 modulation over $50 \mathrm{~m}$ step-index polymer optical fiber (SI-POF) channel at a desired BER level of $1 \times 10^{-3}$ and $1.2 \mathrm{Gbps}$ transmission rate. Furthermore the hardware experiment using commercially available components proves the improvements in the proposed PAM transmission scheme.

Conclusion: The novel PAM transmission scheme is compared to the optimized discrete multi-tone (DMT) transmission with bit-loading. The experimental results show that for a transmission distance less than $50 \mathrm{~m}$ over SI-POF, DMT systems outperform the PAM systems. However, for a SI-POF transmission over the distance longer than $50 \mathrm{~m}$, the proposed scheme can reach a better performance than the DMT systems thanks to the advantage of a lower peak-to-average-power ratio.
\end{abstract}

Keywords: PAM, Single-carrier modulation, DMT, Multi-carrier modulation, POF, PN sequence, Channel estimation, Minimum-phase pre-filter, Reduced-state sequence estimation

\section{Background}

Recently, high-speed transmission over plastic optical fiber (POF) has attracted many research interests $[1,2]$. POF owns the advantages of easier connection and band insensitive, which could be an economic solution for home networking. However, the available transmission bandwidth in POF is limited due to the significant modal dispersion in large core diameter POF. The intensive modal dispersion could be modeled as multi-path channel delay which causes low-pass channel frequency response $[1,2]$. In addition, for different types of short range optical

*Correspondence: mingliu@bjtu.edu.cn

${ }^{2}$ School of Computer and Information Technology, Beijing Jiaotao University, No.3 ShangYuanCun, 100044 Beijing, China

Full list of author information is available at the end of the article communication systems, such as single-mode fiber (SMF), multi-mode fiber (MMF), and optical wireless with visible light communications (VLC), there have similar channel characteristics as POF's, which can be modeled as a low-pass frequency response with very high signal-tonoise ratio (SNR) [3-6]. Therefore, the increasing traffic demands require better bandwidth utilization in the existing short range systems. In the state-of-the-art short range optical transmission systems, the used transmission bandwidth is far wider than the system's $3 \mathrm{~dB}$ bandwidth [3-6]. In order to explore the best transmission performance in these situations, several advanced modulation schemes have been investigated in recent works [7-9].

Multi-carrier modulation (MCM) schemes are widely used for the short range optical transmission systems. The 
discrete multi-tone (DMT) with bit-loading technique can effectively approach the channel capacity. However, MCM schemes have the drawbacks of high peak-toaverage-power ratio (PAPR) and computational complexity $[9,10]$. Compared to the MCM schemes, single carrier modulation (SCM) schemes enjoy the advantages of computational simplicity and low PAPR. The Not-Returnto-Zero (NRZ) coding with equalization offers satisfactory achievable link power budget margin [8,9]. The pulseamplitude modulation (PAM) is another SCM scheme that provides better spectrum efficiency than NRZ coding. As the short range optical transmission systems usually have high SNR, the PAM transmission scheme shows notable advantages over the classical NRZ transmission scheme $[8,11]$.

However, sophisticated equalization techniques are needed for most SCM schemes in order to restore the distorted SCM signal after the transmission over the bandwidth-limited channels [8]. An equalizer is commonly adopted at the receiver side to equalize the received signal. In addition, the decision-feedback equalizer (DFE) is proved to outperform the feed-forward equalizer (FFE) [12]. Moreover, in case of adaptive equalization, long training sequences are required for good convergence, which consequently reduces the overall system efficiency. Moreover, DFE has the drawback of the error propagation which reduces the system performance in the practical implementations. Although some works show that the Tomlinson-Harashima precoding technique can resolve the error propagation problems in DFE [13], it requires a prior information at transmitter side and increases the overall system complexity [13].

Concerning practical PAM transmissions over SI-POF, [14] and [15] summarized available transmission rates with different SCM schemes, such as 1 Gbps over $20 \mathrm{~m}$ SI-POF, $400 \mathrm{Mbps}$ over $50 \mathrm{~m}$ SI-POF, and $170 \mathrm{Mbps}$ over $115 \mathrm{~m} \mathrm{SI-POF}$ in [15] and $10 \mathrm{Mbps}$ over $425 \mathrm{~m}$ SI-POF, $100 \mathrm{Mbps}$ over $275 \mathrm{~m}$ SI-POF and 1 Gbps over $75 \mathrm{~m}$ SI-POF in [14]. Additionally, recent works reported higher transmission rate over $50 \mathrm{~m}$ SI-POF with new designed front-end receivers $[16,17]$. In this work, we focus on a system architecture research of increasing SI-POF transmission rate with existing off-the-shelf components. In order to improve the transmission efficiency of the PAM transmission and avoid the error propagation problem in DFE, we propose a novel pseudo-noise sequence assisted pulse-amplitude modulated (PN-PAM) transmission scheme for short range optical communication systems. This novel scheme is a SCM in nature and is incorporated with a minimum phase pre-filter and a simplified trellis-based equalizer at the receiver side. The coefficients of minimum phase pre-filter could be obtained from a pseudo noise (PN) sequence-based channel estimation with very short training symbol length.
A comparison between the capacities of the novel PAM scheme and the existing DMT scheme over SI-POF is carried out with the same experimental setups. It is shown that this proposed PAM transmission scheme is suitable for other short range optical communication systems due to its advantage of low PAPR.

The remainder of this paper is organized as follows: In "Methods" section, the method of PN-PAM transmission scheme is introduced. In "Theoretical analysis of the PN-PAM transmission" section, a theoretical analysis for PAM transmission over short range step index-POF (SI-POF) system which is modeled as Gaussian low-pass filter. The PAPR of PAM transmission with root-raisedcosine (RRC) filters is also evaluated. In "Numerical analysis of the PN-PAM transmission" section, the proposed PAM transmission scheme is compared with the classical one via simulations. In "Results and discussion" section, a real SI-POF transmission system with commercially available components is used to further prove the merits of the proposed scheme. The comparisons between the PAM and DMT transmissions over practical channels are also presented. Finally, conclusions are highlighted in "Conclusion" section.

\section{Methods}

In optical fiber communication systems, PAM transmission with adaptive DFE has been widely used. However, as the optical fiber channels are quite stable, it is possible to obtain the optimal DFE coefficients with a reduced transmission overhead. Furthermore, as DFE has the drawback of the error propagation, it is necessary to introduce a novel decision mechanism to replace the direct decision in DFE. In this section we propose a novel PAM transmission scheme for optical fiber communications. To achieve satisfactory performance, the reception of this novel scheme includes three main components: the PN sequence based channel estimator, a minimum-phase receiver filter, and a reduced-state sequence estimation(RSSE) based equalizer. More details of the proposed scheme is presented in following part of the section.

\section{PN sequence based channel estimation}

The SCM based transmissions enjoy the advantage of low PAPR over MCM ones and are therefore attractive for optical fiber transmissions. In the meantime they normally require high channel estimation accuracy in order to prevent the error propagation phenomenon in the reception. The PN sequence-based channel estimation was known for its merits of low complexity and high accuracy in wireless communication scenarios [18]. It was recently proved to be very efficient for in DMT transmission over optical fiber [19]. This motivates us to investigate the application of $\mathrm{PN}$ sequence based channel estimation for SCM-based optical fiber transmissions. 
In classical PAM systems, the transmitted symbols are expressed as:

$$
\begin{aligned}
\bar{S}_{\mathrm{PAM}}=\left[\bar{S}_{\mathrm{T}}, \bar{S}_{\mathrm{D}}\right]^{T}=[ & S_{\mathrm{T}}(0), \cdots S_{\mathrm{T}}\left(N_{\mathrm{T}}-1\right), S_{\mathrm{D}}(0), \cdots \\
& \left.S_{\mathrm{D}}\left(N_{\mathrm{D}}-1\right)\right]^{T},
\end{aligned}
$$

where $\bar{S}_{\mathrm{T}}$ is the length- $N_{\mathrm{T}}$ vector of training symbols for the equalizer; $\bar{S}_{\mathrm{D}}$ is the length- $N_{\mathrm{D}}$ vector of PAM data symbols.

When the PN sequence is inserted to the PAM symbols to assist the channel estimation, the transmitted PN-PAM symbols are written as:

$$
\begin{aligned}
\bar{S}_{\mathrm{PN}-\mathrm{PAM}}=\left[\bar{\rho}_{\mathrm{PN}}, \bar{S}_{\mathrm{D}}\right]^{T}= & {\left[\rho_{\mathrm{PN}}(0), \cdots \rho_{\mathrm{PN}}\left(N_{\mathrm{PN}}-1\right),\right.} \\
& \left.S_{\mathrm{D}}(0), \cdots S_{\mathrm{D}}\left(N_{\mathrm{D}}-1\right)\right]^{T},
\end{aligned}
$$

where $\bar{\rho}_{\mathrm{PN}}$ is the vector of PN sequence for channel estimation. The length of $\bar{\rho}_{\mathrm{PN}}$ is $N_{\mathrm{PN}}$.

At the receiver side, the received $\mathrm{PN}$ sequence is used to perform channel estimation. The PN sequence based channel estimation for optical communications has been initially introduced in [19]. The m-sequences are selected as the PN sequences for channel estimations due to their ease of generation and their associated low complexity. The most significant benefit of using $\mathrm{m}$-sequence for channel estimation is its special circular autocorrelation property. The circular autocorrelation of the $\mathrm{m}$-sequence is known as:

$$
\mathrm{CR}_{j}=\frac{1}{N_{\mathrm{PN}}} \sum_{i=0}^{N_{\mathrm{PN}}-1} m_{i} m_{[i+j]_{N_{\mathrm{PN}}}}^{*}=\left\{\begin{array}{cl}
1 & j=0 \\
-\frac{1}{N_{\mathrm{PN}}} & \text { else }
\end{array}\right.
$$

where $m$ is the $\mathrm{m}$-sequence, $(\cdot)^{*}$ is the complex conjugate, $[\cdot]_{N_{\mathrm{PN}}}$ denotes modulo- $N_{\mathrm{PN}}$ operation. With the help of circular autocorrelation property shown in (3), the channel estimation can be simply obtained by performing time domain correlation of known and received $\mathrm{PN}$ sequences:

$$
\widetilde{\bar{h}}=\frac{1}{N_{\mathrm{PN}}} \sum_{i=0}^{N_{\mathrm{PN}}-1}\left(\sum_{l=0}^{N_{\mathrm{H}}-1} h_{l} \rho_{i-l}+w_{i}\right) \cdot m_{[i+j]_{\mathrm{PN}}}^{*}
$$

where $w$ is the noise, $N_{\mathrm{H}}$ is the channel length. In POF channel model, the massive multi-path delay could be modeled as discrete filter taps. Therefore, the maximal channel multi-path delay in real POF channel could be denoted by channel length $N_{\mathrm{H}}$ with number of filter taps.

Finally, the accurate estimate of the channel impulse response (CIR) $\widetilde{\bar{h}}=\left[\widetilde{h}_{0}, \widetilde{h}_{1}, \cdots \widetilde{h}_{N_{\mathrm{H}}-1}\right]^{T}$ can be easily obtained at a very low complexity cost [20]. According to the analysis carried out in [19], the overall complexity of the PN sequence-based channel estimation is $\mathcal{O}\left(N_{\mathrm{PN}}\right.$. $\log N_{\mathrm{PN}}$ ), which is determined by the PN sequence length.

\section{Minimum-phase pre-filtering}

In communication systems, trellis-based equalizer can effectively eliminate the inter-symbol interference (ISI) after transmission over the channel. The maximumlikelihood sequence estimation (MLSE) is recognized as the optimal equalization algorithm in the sense of sequence detection. As the decision is based on a sequence of symbols, it can effectively avoid the error propagation problem of DFE. However, it is worth noting that for PAM with high orders modulations, the computational complexity of MLSE equalizer dramatically increases. The full MLSE equalizer becomes computationally prohibitive when the modulation order is high and/or when the channel length is long. To avoid the prohibitive complexity, a sub-optimal trellis-search based equalizer, namely RSSE, is commonly used for its simplicity in the hardware implementation.

Studies in $[21,22]$ show that, in order to obtain the sub-optimal performance after trellis-based equalization, discrete time minimum-phase overall impulse response needs to be carried out previously. We employ an FFE pre-filter to achieve the minimum-phase overall impulse response. As an accurate CIR is obtained directly from the PN sequence-based channel estimation, it is feasible to calculate the filter coefficients from the estimated CIR. The coefficients of the pre-filter can be calculated in closed-form from the estimated CIR $\widetilde{\bar{h}}$.

In [22], the coefficients of the minimum-phase pre-filter are calculated by the linear prediction from the estimated CIR. The linear prediction is realized by the well-known Levinson-Durbin algorithms. Concretely, the pre-filter is determined as follows:

$$
\widetilde{F}(z)=z^{-N_{\mathrm{H}}} H^{*}\left(1 / z^{*}\right)(1-P(z)),
$$

where $H^{*}\left(1 / z^{*}\right)$ is the time-reversed conjugated CIR, $(1-P(z))$ is the calculated linear prediction filter, $z^{-N_{\mathrm{H}}}$ introduces a delay of $N_{\mathrm{H}}$ samples.

The analysis of this minimum-phase pre-filter calculation shows that the overall computational complexity of linear prediction method is significantly lower than that of the minimum mean-squared error (MMSE)-DFE method [22].

\section{RSSE based equalization}

In contrast to the MLSE equalizer where all possible combinations of symbol sequences are compared with received signal sequence, RSSE dramatically reduces the number of candidates to be compared by applying constellation partitioning and decision-feedback with early decisions [23]. With a proper choice of the number of survivor states, the RSSE based equalizer can approach the optimal performance offered by the MLSE equalizer.

More concretely, the entire symbol alphabet is divided into subsets, and the search trellis is built based on these 
subsets. The subsets need to be chosen such that the Euclidean distance of symbols within each subset is maximized. Once the survival path is determined, the hard decision within the subset is made directly so that only one survival candidate is reserved in each set, while others are simply discarded for the following search. The selection among subsets is not taken in the current step. Therefore, the number of overall trellis states involved in the search is reduced to $Z=\prod_{k=1}^{N_{C}} J_{k}$, where $J_{k}$ is the number of subsets preserved for the symbol $k$ steps before the currently detecting symbol, $N_{\mathrm{C}}$ is the constraint length which is chosen according to the number of significant channel paths, and can be less than the overall channel length $N_{\mathrm{H}}$. It is worth noting that through the choice of $J_{k}$, the performance and complexity of RSSE equalizer can achieve arbitrary trade-off between the optimal MLSE equalizer and the simple DFE equalizer [22]. For example, when $J_{k}=M, 1 \leq k \leq N_{\mathrm{C}}$, the RSSE equalizer becomes the MLSE equalizer for PAM- $M$ modulation. Similarly, when $J_{k}=1,1 \leq k \leq N_{\mathrm{C}}$, since there is only one subset preserved for decision, the RSSE equalizer is turned into a DFE equalizer.

\section{Theoretical analysis of the PN-PAM transmission Gaussian low-pass filter channel model}

In most short range optical transmission systems, the channels show similar features such as a low-pass frequency response and very good channel condition (very high SNR) at low frequency part [3-5]. The Gaussian low-pass filter channel model has been proved to be suitable for the POF systems [24]. In this section, it will be used to model a 50-m SI-POF system for the theoretical investigation of the proposed PN-PAM transmission.

The channel frequency response (CFR) of the Gaussian low-pass filter channel model is written as:

$$
H(f)=A \cdot \exp \left[-\left(\frac{f}{f_{0}}\right)^{2}\right], \quad f_{0}=\frac{f_{3 \mathrm{~dB}}}{\sqrt{\ln (2)}},
$$

where $H(f)$ is the CFR at $f \mathrm{~Hz} ; A$ is the optical fiber loss; $f_{3 \mathrm{~dB}}$ is the $3 \mathrm{~dB}$ bandwidth of the considered 50-m SI-POF system.

\section{PAM transmission over Gaussian low-pass filter channel}

Using the Gaussian low-pass filter channel model introduced in (6), we can theoretically analyze the PAM transmission over a 50-m SI-POF with different equalizers. It is well known that in additive white Gaussian noise (AWGN) channel, channel capacity is calculated as:

$$
C=W \cdot \log _{2}\left(1+\frac{P}{N_{0} W}\right),
$$

where $W$ is the used bandwidth, $P$ is the signal power, $N_{0}$ is the power spectral density of the noise. When signal is transmitted over a multi-path channel, ISI will be introduced among consecutive symbols due to the time dispersion of signal. At the receiver side, noise can be boosted after the equalization depending on the equivalent SNR over the whole signal band. Therefore, the performance of the PAM system can be determined through the corresponding SNR after equalization [25]. Based on the post-equalization SNR, we can derive the achievable rate of the PN-PAM transmission in the Gaussian low-pass filter channel.

As the RSSE equalization used in the proposed scheme is a non-linear process, it is difficult to derive the capacity of the proposed PN-PAM scheme in a straightforward manner. Alternatively, we analytically investigate the PAM transmission system using DFE which is an extreme case of the RSSE equalizer. For PAM transmission system using DFE, we assume that no incorrect decision is fed back. Following the results given in [25], the post-equalization SNR of PN-PAM transmission system is written as:

$$
S N R_{\mathrm{DFE}}=\frac{1-\xi_{\mathrm{DFE}}}{\xi_{\mathrm{DFE}}}
$$

where

$$
\xi_{\mathrm{DFE}}=\exp \left[\frac{T}{2 \pi} \int_{-\pi / T}^{\pi / T} \ln \frac{N_{0}}{H\left(e^{j \omega T}+N_{0}\right)} d \omega\right] .
$$

The integral in the expression of $\xi_{\mathrm{DFE}}$ in (9) can be mathematically approximated by discrete summation. Employing (6), $\xi_{\mathrm{DFE}}$ is estimated by:

$$
\xi_{\mathrm{DFE}} \approx \exp \left[\frac{1}{N_{p}} \sum_{i=1}^{N_{p}} \ln \frac{N_{0} \cdot N_{p}}{A \cdot \exp \left[-\left(\frac{i \cdot \Delta f}{\sqrt{\ln (2)} \cdot f_{\mathrm{ddB}}}\right)^{2}\right]+N_{0} \cdot N_{p}}\right],
$$

where $\Delta f$ is the frequency spacing of the discrete calculation; $N_{p}$ is the number of intervals in order to approximate the integral function. The bandwidth of the PAM system is therefore $W_{\mathrm{PAM}}=\Delta f \cdot N_{p}$. The normalized noise energy within the $\Delta f$ frequency spacing is $N_{0}$.

We assume that the Nyquist bandwidth $(1 / 2 T)$ is used. Introducing the post-equalization SNR in (8) and the channel capacity in (7), we can obtain the capacity of PAM transmission with DFE equalization:

$$
C=2 \cdot W_{\mathrm{PAM}} \cdot \log _{2}\left(1+S N R_{\mathrm{DFE}}\right) .
$$

Introducing the linear approximation method proposed in [26], we can compute the achievable PAM modulation order $b$ as:

$$
b=\frac{S N R(\mathrm{~dB})-A_{2}}{A_{1}},
$$

where $A_{1}$ and $A_{2}$ are coefficients defined in the linear approximation. The exact values of $A_{1}$ and $A_{2}$ for PAM 
modulation can be obtained by following similar manipulations as in [26] for different desired BER levels. The obtained $A_{1}$ and $A_{2}$ are listed in Table 1.

Applying (11) and (12), the available transmission rate can be calculated as:

$$
R_{s}=2 \cdot W_{\mathrm{PAM}} \cdot \frac{10 \cdot \log _{10}\left(\frac{1-\xi_{\mathrm{DFE}}}{\xi_{\mathrm{DFE}}}\right)-A_{2}}{A_{1}} .
$$

Take the 50-m SI-POF transmission system as an example, where the channel model parameters are the same as in [26]. We can get that $f_{3 \mathrm{~dB}}$ is $90 \mathrm{MHz}$ and the measured noise power spectral density is $-113.7 \mathrm{dBm} / \mathrm{Hz}$. We numerically calculate the PAM transmission rate with the frequency spacing $\Delta f$ of $1 \mathrm{MHz}$ and number of intervals $N_{p}$ equal to 1000 . With a target BER of $1 \times 10^{-3}$ (a level that the residual error can be effectively corrected by channel coding), the relationship between PAM transmission rate and used bandwidth is depicted in Fig. 1.

As shown in the figure, the narrower bandwidth, the higher PAM modulation orders. However, concerning transmission rate, there is an optimized used bandwidth and PAM modulation order. For instance, in our POF system model, the optimal used bandwidth in Fig. 1b is around $220 \mathrm{MHz}$. The achievable PAM modulation order with this bandwidth is PAM-8 accordingly in Fig. 1a.

\section{RRC filters for PAM transmissions}

In practical communication systems, the square RRC filters are normally used at the transmitter and receiver in order to reduce the required bandwidth for the transmission. For the RRC filters, a roll-off factor $\beta$ is a measure of the excess bandwidth of the filter, which represents the bandwidth occupied beyond the Nyquist bandwidth of $1 / 2 \mathrm{~T}$. $\beta$ can be chosen between 0 and 1 . Therefore in a practical system, the real required bandwidth $W_{\mathrm{PAM}}$ is denoted as:

$$
W_{\mathrm{PAM}}=\frac{1}{2} R_{s}(1+\beta),
$$

where $R_{S}$ is the PAM symbol rate.

In a pure PAM system without the RRC filter, the PAPR is directly related to the PAM modulation order. However, the use of RRC filter at the transmitter will increase the PAPR with a reduced excess bandwidth or increased filter length [27]. The relationship between the PAPR of the QAM modulation and the RRC filter rolloff factor was studied for PAM-2 and QAM-32 in [27]. Here we investigate the relationship between $\beta$ and PAPR for PAM-2, PAM-4, PAM-8, PAM-16, and PAM-32. The maximal PAPR of the PAM signal after the RRC filtering is evaluated by the following equation:

$$
\mathrm{PAPR}_{\max }=10 \cdot \log _{10}\left(\frac{x_{\max }^{2}}{\mathbb{E}\left[x^{2}\right]}\right),
$$

where $x_{\max }$ is the maximal amplitude of the PAM- $M$ signal. $\mathbb{E}\left[x^{2}\right]$ is the average power of the generated signal. The measured results with a RRC filter length of 8 taps are presented in Fig. 2. From the figure, we can find that the PAPR of the PAM modulated signal after RRC filtering significantly increases when the roll-off factor is lower than 0.4. The PAPR of the PAM-2, PAM- 4 and PAM- 8 with a RRC filter roll-off factor of 0.2 is obtained around 4.3, 6.9 and $8.0 \mathrm{~dB}$, respectively.

\section{Numerical analysis of the PN-PAM transmission}

In this section, we simulate the PN-PAM transmission over the $50-\mathrm{m} \mathrm{SI-POF} \mathrm{system.} \mathrm{The} \mathrm{modulation} \mathrm{is} \mathrm{cho-}$ sen to be PAM- 8 which has been shown to be the best modulation scheme for the given channel condition. The symbol rate is set to be 440 Mega symbols per second. Therefore the theoretically minimal required bandwidth is $220 \mathrm{MHz}$ when roff-off factor in RRC filter is assumed at 0 . In the practical simulation system, we use a square RRC transceiver filter to reduce the used bandwidth of the PAM transmissions. The roll-off factor is set to be 0.2. Therefore, the actual used bandwidth in simulations is $264 \mathrm{MHz}$. The estimated SNR with the noise PSD of $-113.7 \mathrm{dBm} / \mathrm{Hz}$ is about $29.5 \mathrm{~dB}$.

\section{PN-PAM transmission with different equalization methods}

Firstly, in Fig. 3, we present the simulation results of PAM-8 transmission over the 50-m SI-POF channel with different equalization methods. For the classical adaptive DFE equalizations, two typical algorithms referred to as recursive least square (RLS) and least mean square (LMS) are adopted. In genie-aided mode, we initially transmit 10,000 training symbols to get the DFE coefficients and subsequently using the known symbols as the decided symbols for the DFE feedback. Alternatively, in directdecision mode, we use decided symbols for the DFE feedback. In both cases, the number of decision feedback taps is 6 and the number of FFE taps is 24. The simulation results show that DFE-RLS and DFE-LMS provide similar performance in the 50-m SI-POF system with PAM-8 transmission. However, in both DFE-RLS and DFE-LMS schemes, due to the error propagation in direct-decision

Table 1 Parameters for the relationship between SNR and available PAM modulation order with different desired BER

\begin{tabular}{llllllll}
\hline BER & $10^{-1}$ & $10^{-2}$ & $10^{-3}$ & $10^{-4}$ & $10^{-5}$ & $10^{-6}$ & $10^{-7}$ \\
\hline$A_{1}$ & 4.926 & 5.848 & 6.020 & 6.090 & 6.120 & 6.150 & 6.156 \\
$A_{2}$ & -5.307 & -1.087 & 1.214 & 2.741 & 3.913 & 4.814 & 5.620 \\
\hline
\end{tabular}




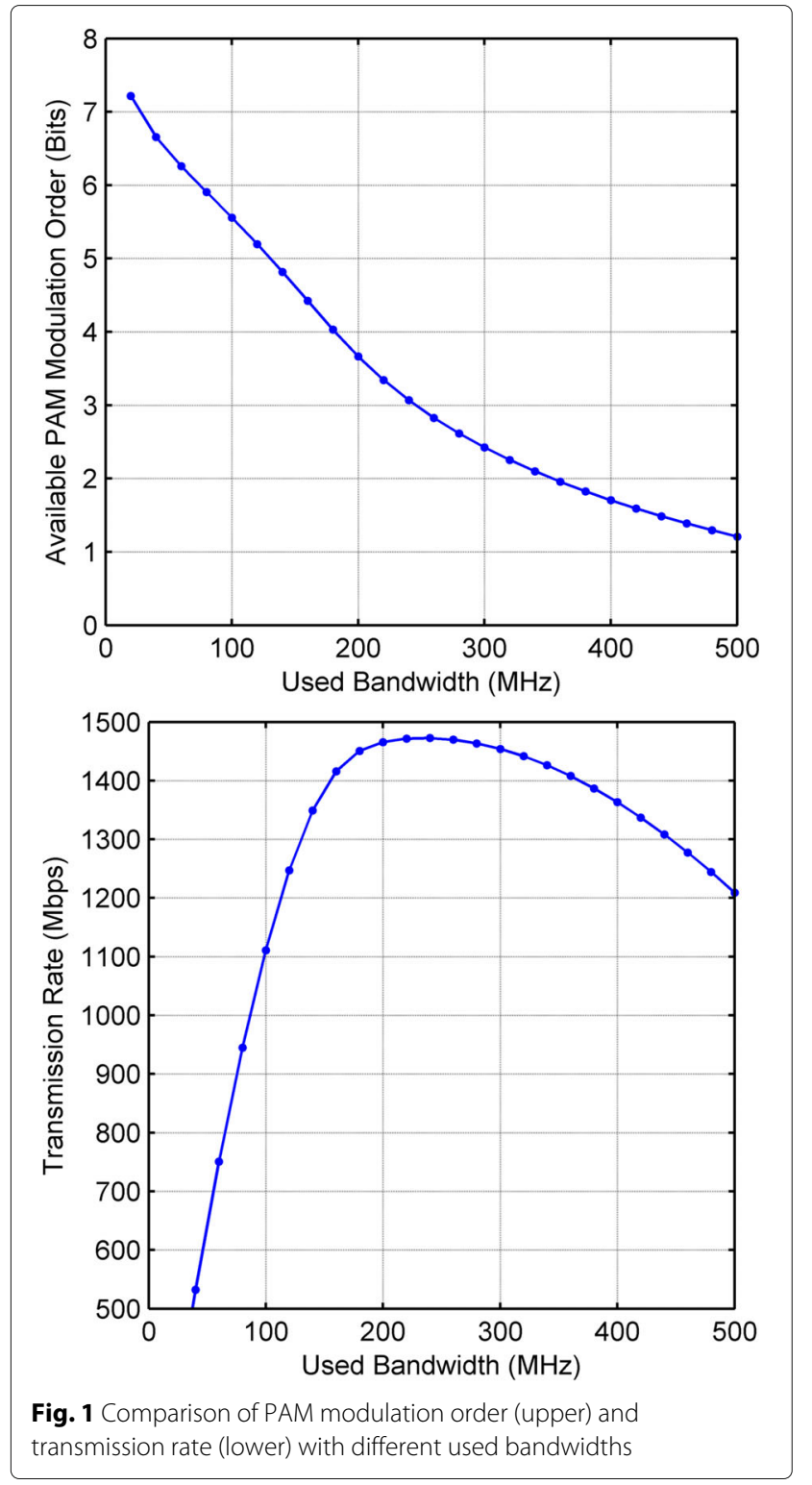

mode, there is an approximate $1.5 \mathrm{~dB}$ degradation at the BER level of $1 \times 10^{-3}$ compared to the genie-aided mode. Therefore it is crucial to avoid the error propagation problem in the practical systems.

Then we simulate the proposed PN-PAM transmission scheme with RSSE. The PN sequence length for channel estimation is set to be 255 symbols and a cyclic-prefix with the length of 33 symbols is added to the PN sequence in order to prevent the ISI from previous transmitted symbols. The length of the estimated CIR is 7 taps. The first 6 most significant taps are used to calculate the minimum-phase pre-filter. An illustration of the multipath CIR in original Gaussian low-pass filter channel model and after pre-filtering is presented in Fig. 4. As shown in the figure, a main path is recovered at the first

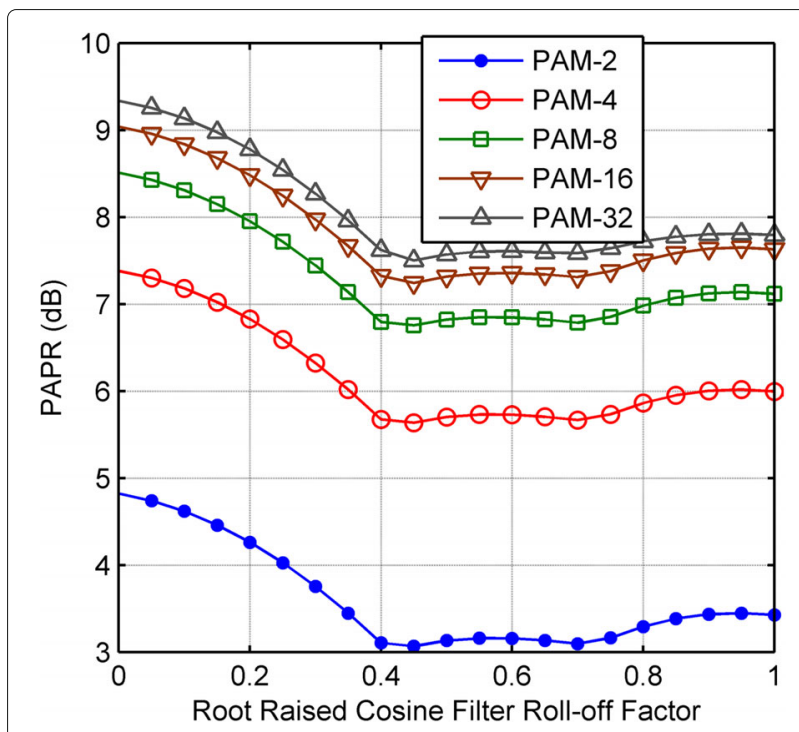

Fig. 2 Relationship between the PAM modulation order and RRC filter roll-off factor

tap after pre-filtering. Then the most significant multipath component is located at the second tap, which owns an amplitude about 0.86 . Therefore, we design and compare two RSSE structures in 50-m POF transmissions. The first RSSE structure uses the first 2 most significant delay taps for symbols decision. Hence the length of the RSSE trellis is selected to 2 . The number of subsets for the first delay $\operatorname{tap} J_{1}$ is set to 2 and the number of subsets for the second delay tap $J_{2}$ is set to 1 . The second RSSE structure uses all of the 5 delay taps for symbols decision. For each delay tap, the number of subsets $J_{k}$ is set to 1 . In the second RSSE structure, as each delay tap only has one subset, the RSSE equalizer turns into a DFE equalizer with 5 taps. Obviously, for both of the RSSE structure, the overall complexity is very low.

After simulations of the transmission over 50-m SIPOF channel, performances of RSSE equalizers are depicted in Fig. 3. As been shown in the figure, the RSSE $(2,1)$ equalizer can achieve similar performance to classical DFE equalizers in genie-aided mode, which has $1.5 \mathrm{~dB}$ improvement with the practical DFE equalizers in direct-decision mode. Additionally, the RSSE $(1,1,1,1,1)$ equalizer has a very close performance to classical DFE equalizers. Therefore, it is clear that in the 50-m SI-POF channel, using $\operatorname{RSSE}(2,1)$ equalizer can solve the error propagation problem in classical DFE equalizers.

\section{PAM transmission with respect to different training sequence lengths}

In addition, as mentioned in "Methods" section, the novel PAM transmission scheme leads to an improved 


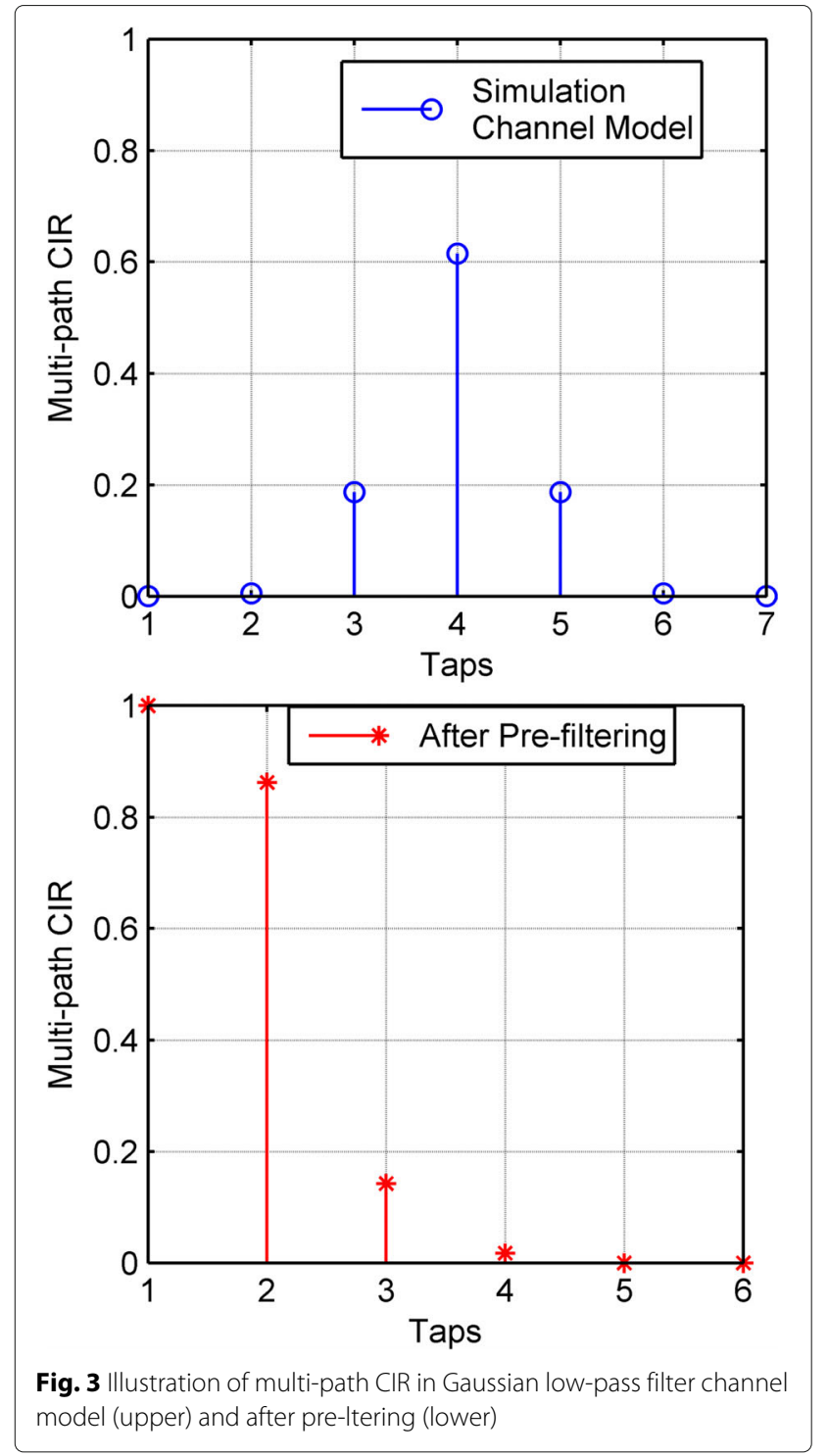

transmission efficiency. The training sequence takes up a certain amount of transmission energy and hence reduces the overall system efficiency. For the classical DFE-RLS and DFE-LMS equalizers, the predefined training sequence is inserted before the data symbols. The equalizers work in training mode when the training symbols are received and then switch to the equalization mode when data symbols are received. In equalization mode, there are two feed-back methods. In general transmissions, the feed-back bits are obtained from decisions, which is called as direct-decision mode. However, in this mode, decision error will be propagated and cause penalties. Besides, it is also possible to use known pilot bits as the feed-back bits. In this mode, error propagation could be avoided due to the known pilot bits at receiver. This mode is called as genie-aided mode. In practical system, the known pilot bits cannot be used to transmit

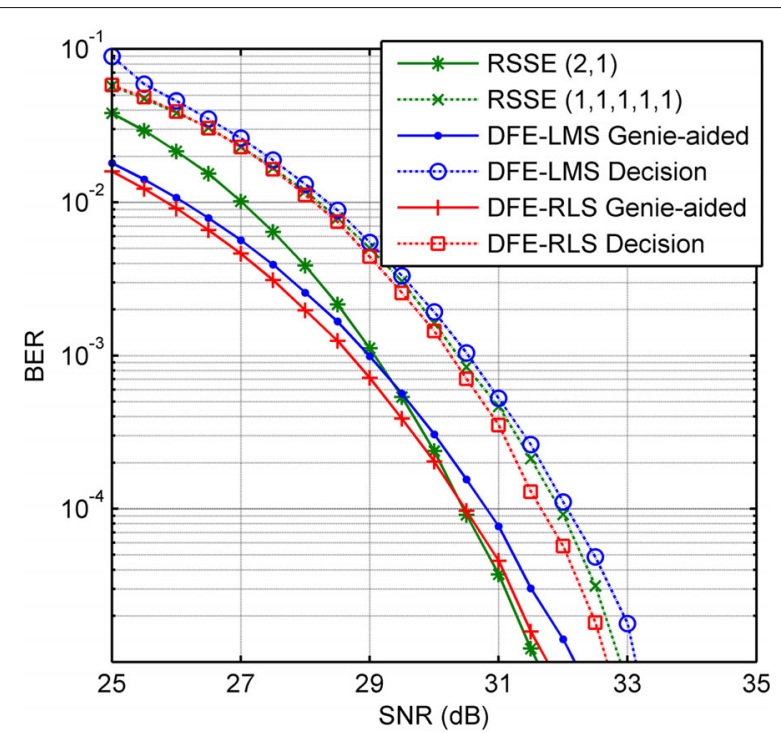

Fig. 4 Simulation results of the PAM transmission over 50 meters SI-POF with different equalization methods

message. Therefore, the genie-aided mode is only used to theoretical optimal performance evaluations.

For the proposed scheme, the training sequence is the PN sequence inserted before the data symbols. We simulate the PAM-8 transmission over the 50-m SI-POF channel with different training sequence lengths for both classical DFE and the proposed scheme. The SNR is fixed to $29.5 \mathrm{~dB}$. The BER performance is depicted in Fig. 5 .

With a PN sequence length longer than 255 symbols, the proposed system achieves the best BER performance of around $4.6 \times 10^{-4}$. However, the classical DFE based

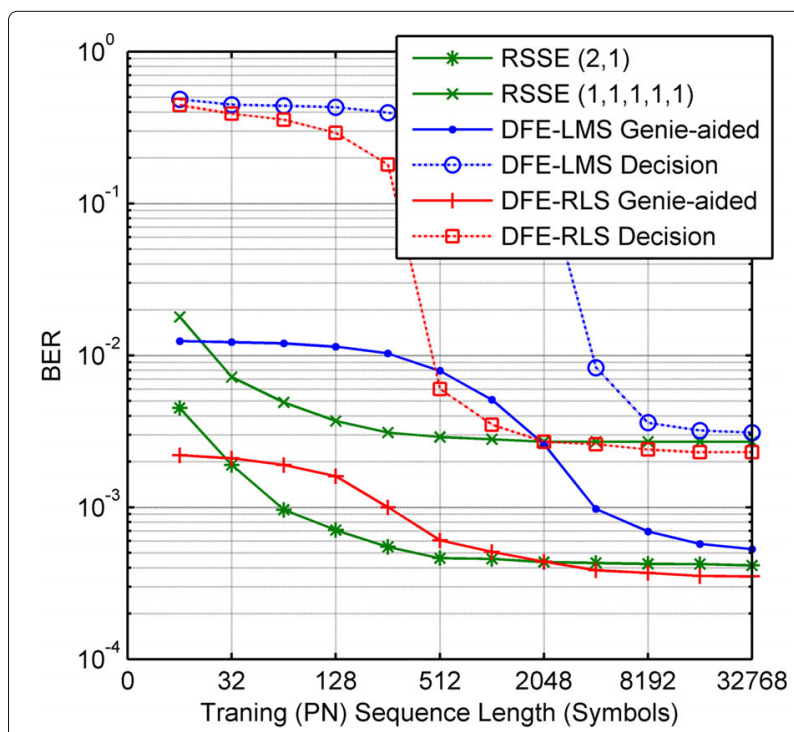

Fig. 5 Simulation results of the PN-PAM transmission over 50-m SI-POF with different training sequence lengths 
equalizers require much longer training sequences. Simulation results show that even in the genie-aided mode, in order to get converged BER performance, the DFERLS requires a training sequence longer than 4096 symbols and the DFE-LMS requires a training sequence longer than 32,768 symbols. Moreover, when DFE works in direct-decision mode, even extremely long training sequence is adopted, they can only reach the best BER performance of around $2.0 \times 10^{-3}$.

\section{Summary}

From simulations shown above, it is obvious that the proposed PN-PAM transmission scheme can achieve higher system efficiency. The improvement comes from two aspects. On the one hand, the PN-based channel estimation requires very little training overhead, roughly 0.01 to 0.05 compared to the classical DFE schemes. On the other hand, the RSSE equalization leads to an improved BER performance with the help of the reduced error propagation compared to classical DFE schemes.

\section{Results and discussion}

In order to verify the proposed PAM transmission schemes, we setup a practical system for experimental comparisons. We also compared PAM transmissions to DMT transmissions with different POF lengths.

\section{Experimental system setups}

In contrast to most experimental POF transmission systems, we use a commercially available digital to analog convertor (DAC) and analog to digital convertor (ADC). Both DAC and ADC are provided from Texas Instruments. The DAC (DAC5681) has 1 Giga samples per second sampling rate and 16 bits resolution. The ADC (ADC12D1800RFRB) has 1.8 Giga samples per second sampling rate and 12 bits resolution. In order to drive the resonant-cavity light emitting diode (RCLED), we designed an amplifying circuit at the transmitter using a commercially available amplifier (OPA695) also from Texas Instruments. The designed transmission bandwidth is $250 \mathrm{MHz}$.

At the transmitter, RCLED is provided from Firecomms (FC300R-120) with a $3 \mathrm{~dB}$ bandwidth of $100 \mathrm{MHz}$. The biasing current is $20 \mathrm{~mA}$ and the coupled power into the SI-POF is $-0.2 \mathrm{dBm}$. PMMA $\Phi 1 \mathrm{~mm}$ SI-POFs (Eska MEGA) are prepared with different lengths $(15,30,50,75$ and $100 \mathrm{~m})$. At the receiver, a photodiode combined with a trans-impedance amplifier (FC-1000D-120) is employed to detect the received optical signal. The $3 \mathrm{~dB}$ bandwidth of the receiver is $625 \mathrm{MHz}$. The block diagram of the experimental system is presented in Fig. 6.

Both the novel PN-PAM transmission scheme and classical PAM transmission scheme is generated off-line in computer. For the novel PN-PAM transmission scheme, the PN length is set to be 255 symbols and for classical PAM transmission scheme, the training sequence length is chosen as 5000 symbols. The roll-off factor of the RRC filter is selected as 0.25 . Therefore, PAM symbol rate is set to 400 Mega symbols per second. The total used bandwidth is $400 \div 2 \times 1.25=250 \mathrm{MHz}$. As 2 times oversampling is adopted at the transmitter, the DAC sampling rate is 800 Mega samples per second. The ADC works at 1.8 Giga samples per second. At the receiver, for classical PAM transmission with DFE, the number of decision feedback taps is 6 and the number of FFE taps is 24. For the proposed PN-PAM transmission scheme, the first 13 prominent taps in the CIR estimate are used for the calculation of minimum-phase pre-filter. The performance is evaluated with 20 frames, each containing 14,500 symbols. Therefore, in our experimental systems, the overall training overhead is approximately $25.6 \%$ for PAM with DFE (5000 training symbols, 14,500 data symbols) and $1.7 \%$ for the proposed PAM transmission scheme (255 PN symbols, 14,500 data symbols).

In the meantime, the DMT transmission signals are also generated for the comparison. For the DMT system, we setup the system with the same $250 \mathrm{MHz}$ used bandwidth

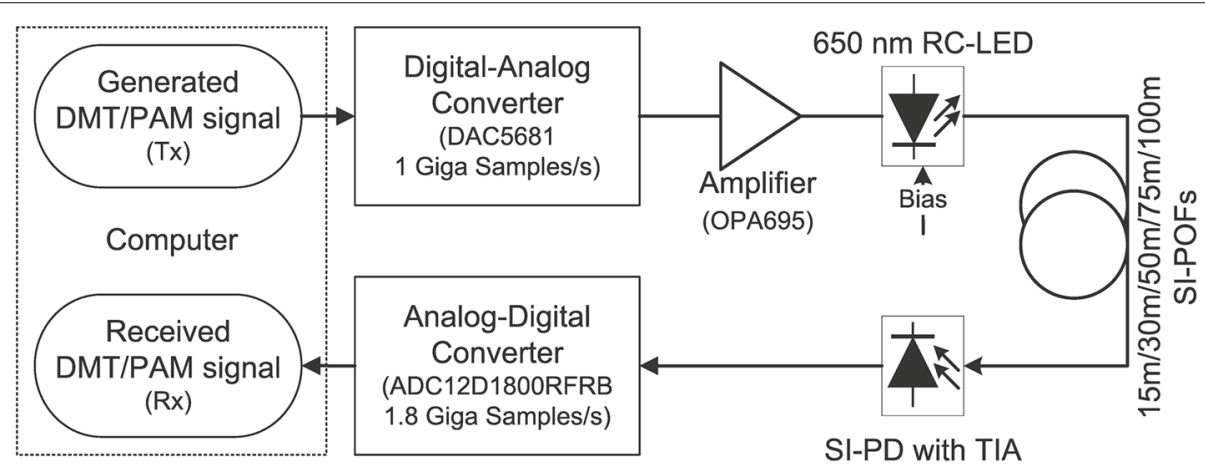

Fig. 6 Experimental setup 
as the PAM system. The DAC sampling rate is set to 1 Giga samples per second indicating 4 times oversampling. The number of available subcarrier is 512 . The first subcarrier is closed to avoid the direct current component. Bit-loading algorithm with linear approximation [26] is employed to allocate bits and power to each subcarrier. The DMT signal is digitally clipped with an optimal clipping ratio of $10 \mathrm{~dB}$. The hybrid pseudo-noise and zeropadding DMT scheme [19] is adopted for its advantages compared to the classical DMT scheme. The PN sequence length is 255 symbols and the ZP length is 32 symbols. The ADC works at 1.8 Giga samples per second. The performance is evaluated with 20 frames, each consisting of 7 DMT symbols.

\section{Experimental results}

For the proposed PN-PAM transmission scheme and DMT transmission scheme, we can estimate channel response using the received $\mathrm{PN}$ sequence. The estimated CIR of 15, 30, 50, 75 and $100 \mathrm{~m}$ SI-POF for the PAM transmissions are presented in Fig. 7. As shown in the figure, the CIRs exhibit significant time dispersion which causes ISI among consecutive symbols. In addition, the system overall non-linearity causes multi-path delay spreading, which is shown as the additional delay path at $9^{\text {th }}$ and $10^{\text {th }}$ taps in Fig. 7. Based on the CIR, we can calculate the coefficients of the minimum-phase pre-filter using (5). The after pre-filtering are presented in Fig. 8. As we can see from the figure, the normalized impulse responses have significantly reduced the pre-interference before the main path. This is essential for the subsequent DFE or RSSE equalizations.

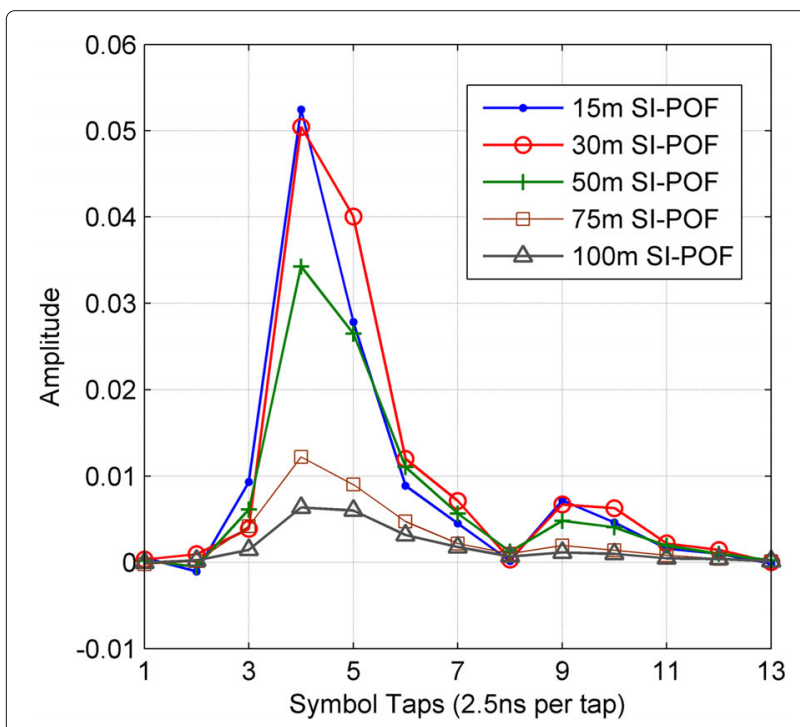

Fig. 7 Channel impulse response of 15, 30, 50, 75 and $100 \mathrm{~m}$ SI-POF transmission

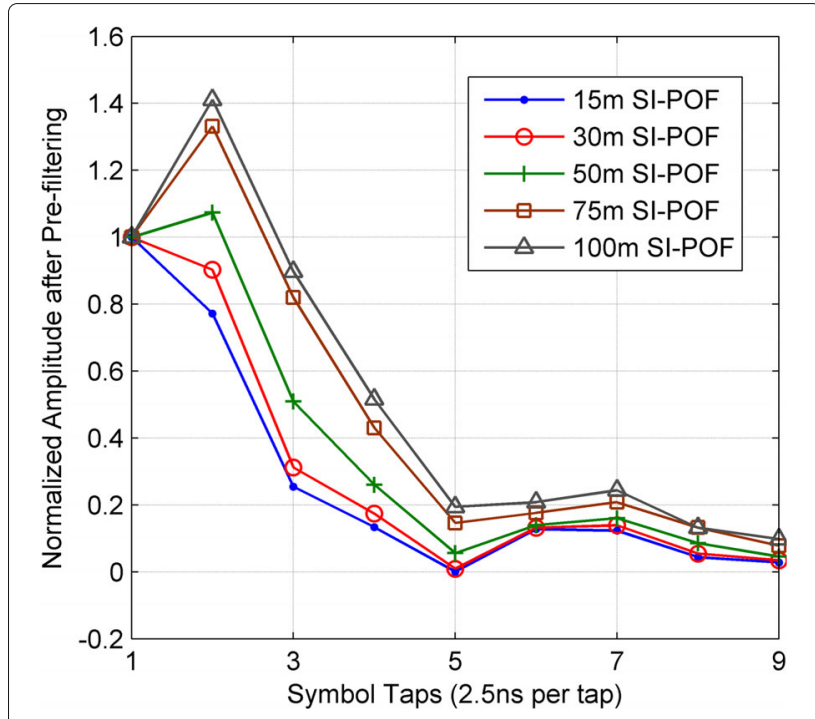

Fig. 8 Normalized impulse responses of 15, 30, 50, 75 and $100 \mathrm{~m}$ SI-POF transmission after pre-filtering

According to the channel conditions, we evaluate several PAM modulations for each transmission length. Both classical PAM with DFE scheme and the proposed PNPAM transmission scheme are tested. The comparison of the BER performances is presented in Fig. 9. In addition, the BER performance of PAM- 4 transmission over 15, 30 and $50 \mathrm{~m}$ SI-POF, the PAM-2 transmission over 15, 30, 50,75 and $100 \mathrm{~m} \mathrm{SI-POF}$ is measured with error-free (i.e., $\left.\mathrm{BER}<1 \times 10^{-6}\right)$. Therefore, these results are not depicted in Fig. 9. For PAM-8 transmissions, the proposed PNPAM scheme can achieve the BER performance of around

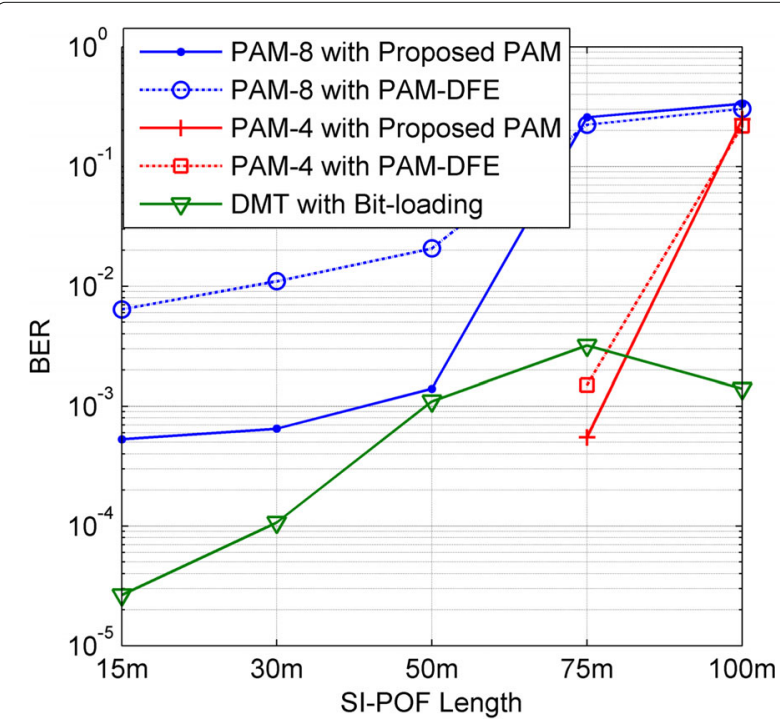

Fig. 9 Experimental comparisons of PN-PAM and DMT transmission over different length of SI-POFs 
$1 \times 10^{-3}$ over 15,30 and $50 \mathrm{~m}$ SI-POF. However, for classical PAM with DFE, the BER performance is only around $1 \times 10^{-2}$ with the same conditions. This BER degradation is mainly due to the error propagation when DFE switches to the direct-decision mode after 5000 training symbols. In addition, it is worth noting that in the proposed PAM scheme, the length of the training sequence is only 255 symbols.

In summary, it can be concluded that using the proposed PN-PAM transmission scheme, PAM-8 is suitable for 15, 30 and $50 \mathrm{~m}$ SI-POF transmission with a desired BER around $1 \times 10^{-3}$. As the symbol rate of the PAM transmission is 400 Mega symbols per second, the total achievable bit rate is 1.2 Gbps. For 75 m SI-POF, PAM4 is a reasonable choice and $800 \mathrm{Mbps}$ can be achieved. Finally, PAM-2 can support a quasi-error-free transmission $\left(\mathrm{BER}<1 \times 10^{-6}\right)$ over $100 \mathrm{~m}$ SI-POF, which leads to a transmission rate of $400 \mathrm{Mbps}$.

\section{Comparison and discussion}

In this section, we introduce the DMT transmissions for comparisons. In order to implement a fair comparison with the PAM transmissions, we employ bit-loading algorithms and allocate bits for total transmission rates of the DMT system at 1.2 Gbps over 15,30 , and $50 \mathrm{~m} \mathrm{SI-}$ POF, 800 Mbps over $75 \mathrm{~m}$ SI-POF and 400 Mbps over 100 $\mathrm{m}$ SI-POF.

The BER performances of DMT are also depicted in Fig. 9 and a summary of experimental results in different transmission schemes with fixed transmission bit rate is presented in Table 2. As shown in the results, we can find that the proposed PN-PAM transmission scheme achieves similar performance to the DMT transmission in the $50-\mathrm{m}$ SI-POF system.

In addition, for the transmission length shorter than $50 \mathrm{~m}$, the DMT systems present better performance. This is mainly due to the fact that for transmission lengths shorter than $50 \mathrm{~m}$, due to the high SNR at receiver, DMT can fully approach the channel capacity with the help of bit-loading. However, when high order PAM modulations are employed for short range communications, the transmitter non-linearity distortion will seriously affect overall performance.
Furthermore, for the transmission lengths longer than $50 \mathrm{~m}$, PN-PAM transmission systems achieve better performance. From the aforementioned PAPR results in Fig. 2, we can find that PAM-2 and PAM-4 present lower PAPR than DMT with $10 \mathrm{~dB}$ clipping. Therefore, with the fixed DAC output dynamic range, the PN-PAM-based transmission systems can achieve higher transmitting power and obtain better performance.

\section{Conclusion}

In this paper, we introduced a novel PAM transmission scheme for short range optical transmission systems. The novel PN-PAM transmission scheme includes PN sequence-based channel estimation, minimum-phase receiver filter and RSSE based equalizer. A theoretical analysis for the PN-PAM transmission over Gaussian low-pass frequency response channel was also presented. From the theoretical analysis, the PAM- 8 modulation is proved to be the best modulation choice for the $50-\mathrm{m}$ SI-POF system.

The simulation results show that, compared to the classical PAM transmission with DFE equalization, the proposed PN-PAM transmission scheme can totally achieve $1.5 \mathrm{~dB}$ gain in a $50-\mathrm{m}$ SI-POF system with an affordable complexity increase. Furthermore, simulation results reveal that the proposed PAM scheme requires less than 0.01 to 0.05 cost of system training symbol overhead compared to the classical DFE schemes, and can largely increase overall system efficiency.

Experimental systems with commercially available components are also investigated for both PN-PAM and DMT transmissions with the same used bandwidths. Experimental results show that, for the SI-POF lengths longer than $50 \mathrm{~m}$, the proposed PN-PAM transmission scheme outperforms the classical PAM transmission with DFE equalization as well as the DMT transmissions that has been optimized in a previous work [19]. In the meantime, for the transmission lengths shorter than $50 \mathrm{~m}$, the proposed PN-PAM transmission scheme performs better than the classical PAM with DFE equalization but worse than the DMT transmissions. This degradation can be understood by the fact that in order to achieve the same transmission rate as DMT, the PN-PAM based

Table 2 Summary of experimental results in different transmission schemes with fixed transmission bit rate

\begin{tabular}{lllll}
\hline \multirow{2}{*}{ POF Length } & Bit Rate & BER & & \\
\cline { 3 - 4 } & & Proposed PAM & $6.4 \times 10^{-3}$ & DAM with DFE \\
\hline $15 \mathrm{~m}$ & $1.2 \mathrm{Gbps}$ & $5.3 \times 10^{-4}$ & $1.1 \times 10^{-2}$ & $2.7 \times 10^{-5}$ \\
$30 \mathrm{~m}$ & $1.2 \mathrm{Gbps}$ & $6.3 \times 10^{-4}$ & $2.1 \times 10^{-2}$ & $1.1 \times 10^{-4}$ \\
$50 \mathrm{~m}$ & $1.2 \mathrm{Gbps}$ & $1.4 \times 10^{-3}$ & $1.5 \times 10^{-3}$ & $1.1 \times 10^{-3}$ \\
$75 \mathrm{~m}$ & $800 \mathrm{Mbps}$ & $5.5 \times 10^{-4}$ & $<10^{-6}$ & $3.2 \times 10^{-3}$ \\
$100 \mathrm{~m}$ & $400 \mathrm{Mbps}$ & $<10^{-6}$ & $1.4 \times 10^{-3}$ \\
\hline
\end{tabular}


systems need to employ higher order modulations when the channel condition is good. However, the higher order modulations are more vulnerable to the non-linearity of the SI-POF system.

Therefore, it can be concluded that the proposed PNPAM transmission scheme outperforms the classical PAM with DFE equalization in terms of both BER performance and overall system throughput. The complexity increase due to the use of RSSE equalizer is also acceptable. For short range optical transmissions with POF length longer than $50 \mathrm{~m}$, the proposed PN-PAM transmission scheme is a better choice than the DMT transmission scheme.

\begin{abstract}
Abbreviations
ADC: Analog to digital convertor; AWGN: Additive white Gaussian noise; CFR: Channel frequency response; CIR: Channel impulse response; DFE: Decision feedback equalizer; DAC: Digital to analog convertor; DMT: Discrete multi-tone; FFE: Feed-forward equalizer; ISI: Inter-symbol interference; LMS: Least mean square; MCM: Multi-carrier modulation; MLSE: Maximumlikelihood sequence estimation; MMF: Multi-mode fiber; MMSE: Minimum mean-squared error; NRZ: Not-return-to-zero; PAM: Pulse-amplitude modulated; PAPR: Peak-to-average-power ratio; PN: Pseudo-noise; PN-PAM: Pseudo-noise sequence assisted pulse-amplitude modulated; RCLED: Resonant-cavity light emitting diode; RLS: Recursive least square; RRC: Root-raised-cosine; RSSE: Reduced-state sequence estimation; SCM: Single carrier modulation; SI-POF: Step-index polymer optical fiber; SMF: Single-mode fiber; SNR: Signal-to-noise ratio; VLC: Visible light communications
\end{abstract}

\section{Acknowledgements}

Not applicable

\section{Funding}

Peng's work is supported by the National Natural Science Foundation of China (Grant No. 61601114) and the Natural Science Foundation of Jiangsu Province (BK20160692). Liu's work is supported by the National Natural Science Foundation of China (Grant No. 61501022) and the Fundamental Research Funds for the Central Universities (2017JBM028).

\section{Availability of data and materials}

Not applicable

\section{Authors' contributions}

LP contributed to entire studies of this work, manuscript preparation and manuscript editing. ML contributed to system design, manuscript revision and manuscript editing. MH contributed to theoretical studies and manuscript revision. SH contributed to experimental studies and manuscript revision. All authors read and approved the final manuscript.

\section{Authors' information}

Linning Peng received his PhD degrees from IETR (Electronics and Telecommunications Institute of Rennes) laboratory at INSA (National Institute of Applied Sciences) of Rennes, France, in 2014. From 2014, he is a research associate at Southeast University. His research interests are in design and optimization for communication systems

Ming Liu received the B.Eng. and M.Eng. degrees from the Xi'an Jiaotong University, China, in 2004 and 2007, respectively, and the Ph.D. degree from the National Institute of Applied Sciences (INSA), Rennes, France, in 2011, all in electrical engineering. He was with the Institute of Electronics and Telecommunications of Rennes (IETR) as a postdoctoral researcher from 2011 to 2015. He is now with Beijing Jiaotong University, China, as an Associate Professor. His main research interests include multicarrier transmissions, MIMO techniques, space-time coding and Turbo receiver.

Maryline Hélard received the M.Sc and PhD degrees from INSA (National Institute of Applied Sciences) of Rennes and the Habilitation degree from Rennes 1 University in 1981, 1884 and 2004 respectively. In 1985, she joined
France Telecom Research Laboratory as a research engineer and since 1991 she carried out physical layer studies in the field of digital television and wireless communications. In 2007, she joined INSA as a professor and she is now the co-director of the Communication Department at IETR (Electronics and Telecommunications Institute of Rennes). She is co-author of 22 patents and several papers (Journal and conferences). Her current research interests are in the areas of digital communications such as equalization, synchronization, iterative processing, OFDM, MC-CDMA, channel estimation, and MIMO techniques applied to wireless communications and more recently to wire communications (ADSL, optical).

Sylvain Haese received the engineer and PhD degrees in electrical engineering from INSA (National Institute of Applied Sciences) Rennes, France, in 1983 and 1997 respectively. From 1984 to 1993 he was an analog IC designer for automotive and RF circuits. In 1993, he joined INSA and carried out research activity at IETR laboratory where he conducted research for automotive powerline applications and for RF wideband channel sounder circuitry. He is currently involved in hardware analog implementation for RF and optical circuits with the Communication Department at IETR (Electronics and Telecommunications Institute of Rennes).

Ethics approval and consent to participate

Not applicable

Consent for publication

Not applicable

Competing interests

The authors declare that they have no competing interests.

\section{Publisher's Note}

Springer Nature remains neutral with regard to jurisdictional claims in published maps and institutional affiliations.

\section{Author details}

${ }^{1}$ Institute of Information Science and Engineering, Southeast University, No.2 SiPaiLou, 210096 Nanjing, China. ${ }^{2}$ School of Computer and Information Technology, Beijing Jiaotao University, No.3 ShangYuanCun, 100044 Beijing, China. ${ }^{3}$ IETR (Institute of Electronic and Telecommunications in Rennes), INSA-Rennes (Institut National des Sciences Appliquées de Rennes), 20 Avenue des Buttes de Coësmes, 35708 Rennes, France.

Received: 9 April 2017 Accepted: 1 August 2017

Published online: 04 September 2017

\section{References}

1. Okonkwo, CM, Tangdiongga, E, Yang, H, Visani, D, Loquai, S, Kruglov, R, Charbonnier, B, Ouzzif, M, Greiss, I, Ziemann, O, Gaudino, R, Koonen, AMJ: Recent Results from the EU POF-PLUS Project: Multi-Gigabit Transmission over $1 \mathrm{~mm}$ Core Diameter Plastic Optical Fibers. IEEE/OSA J. Light. Tech. 29(2), 186-193 (2011)

2. Popov, M: Recent Progress in Optical Access and Home Networks: Results from the ALPHA Project. In: Proc. ECOC. IEEE, Geneva, (2011)

3. Kai, Y, Nishihara, M, Tanaka, T, Takahara, T, Lei, L, Zhenning, T, Bo, L, Rasmussen, JC, Drenski, T: Experimental comparison of pulse amplitude modulation (PAM) and discrete multi-tone (DMT) for short-reach 400-Gbps data communication. In: Proc. ECOC. IEEE, London, (2013)

4. Lee, SCJ, Breyer, F, Randel, S, Cardenas, D, van den Boom, HPA, Koonen, AMJ: Discrete multitone modulation for high-speed data transmission over multimode fibers using 850-nm VCSEL. In: Proc. OSA/OFC/NFOEC. IEEE, USA, (2009)

5. Kottke, C, Hilt, J, Habel, K, Vucic, J, Langer, KD: 1.25 Gbit/s visible light WDM link based on DMT modulation of a single RGB LED luminary. In: Proc. ECOC. IEEE, Amsterdam, (2012)

6. Joncic, M, Kruglov, R, Haupt, M, Caspary, R, Vinogradov, J, Fischer, UHP: Four-Channel WDM Transmission Over 50 m SI-POF at 14.77 Gb/s Using DMT Modulation. IEEE Photonics. Tech. Letters. 26(13), 1328-1331 (2014)

7. Randel, S, Breyer, F, Lee, SCJ, Walewski, JW: Advanced Modulation Schemes for Short-Range Optical Communications. IEEE Sel. Top. Quant. Electron. 16(5), 1280-1289 (2010)

8. Loquai, S, Kruglov, R, Schmauss, B, Bunge, C-A, Winkler, F, Ziemann, O, Hartl, E, Kupfer, T: Comparison of Modulation Schemes for $10.7 \mathrm{~Gb} / \mathrm{s}$ 
Transmission Over Large-Core 1 mm PMMA Polymer Optical Fiber. IEEE/OSA J. Light. Tech. 31(13), 2170-2176 (2013)

9. Schmogrow, R, Winter, M, Meyer, M, Hillerkuss, D, Wolf, S, Baeuerle, B, Ludwig, A, Nebendahl, B, Ben-Ezra, S, Meyer, J, Dreschmann, M, Huebner, M, Becker, J, Koos, C, Freude, W, Leuthold, J: Real-time Nyquist pulse generation beyond $100 \mathrm{Gbit} / \mathrm{s}$ and its relation to OFDM. Optics Express. 20(1), 317-337 (2012)

10. Armstrong, J: OFDM for Optical Communications. IEEE/OSA J. Light. Tech. 27(3), 189-204 (2009)

11. Szczerba, K, Westbergh, $\mathrm{P}$, Agrell, E, Karlsson, M, Andrekson, PA, Larsson, A: Comparison of Inter symbol Interference Power Penalties for OOK and 4-PAM in Short-Range Optical Links. IEEE/OSA J. Light. Tech. 31(22), 3525-3534 (2013)

12. Zeolla, D, Antonino, A, Bosco, G, Gaudino, R: DFE Versus MLSE Electronic Equalization for Gigabit/s SI-POF Transmission Systems. IEEE Photon. Technol. Lett. 23(8), 510-512 (2011)

13. Rath, R, Rosenkranz, W: Tomlinson-Harashima Precoding for Fiber-Optic Communication Systems. In: Proc. of ECOC. IEEE, London, (2013)

14. Straullu, S, Abrate, S: Overview of the performances of PMMA-SI-POF communication systems. In: Proc. SPIE 8645, Broadband Access Communication Technologies VII. SPIE, San Francisco, (2013)

15. Atef, M, Zimmermann, H: Optical Communication over Plastic Optical Fibers, Springer Series in Optical Sciences, Vol. 172. Springer-Verlag, New York (2013)

16. Gimeno, C, Guerrero, E, Sanchez-Azqueta, C, Royo, G, Aldea, C, Celma S: A new equalizer for $2 \mathrm{~Gb} / \mathrm{s}$ short-reach SI-POF links. In: Proc. SPIE 9520, Integrated Photonics: Materials, Devices, and Applications III. SPIE, Barcelona, (2015)

17. Gimeno, C, Guerrero, E, Sanchez-Azqueta, C, Aguirre, J, Aldea, C, Celma, S: Multi-Rate Adaptive Equalizer for Transmission Over Up to 50-m SI-POF. IEEE Photon. Technol. Lett. 29(7), 587-590 (2017)

18. Song, J, Yang, Z, Yang, L, Gong, K, Pan, C, Wang, J, Wu, Y: Technical Review on Chinese Digital Terrestrial Television Broadcasting Standard and Measurements on Some Working Modes. IEEE Trans. Broadcast. 53(1), 1-7 (2007)

19. Peng, L, Hélard, M, Haese, S, Liu, M, Hélard, J-F: Hybrid PN-ZP-DMT Scheme for Spectrum-Efficient Optical Communications and Its Application to SI-POF. IEEE/OSA J. Light. Technol. 32(18), 3149-3160 (2014)

20. Liu, M, Crussière, M, Hélard, J-F: Improved Channel Estimation Methods based on PN sequence for TDS-OFDM. In: Proc. International Conference on Telecommunications (ICT). Jounieh, (2012)

21. Gerstackerand, WH, Schober, R: Equalization Concepts for EDGE. IEEE Trans. Wirel. Commun. 1(1), 190-199 (2002)

22. Gerstacker, WH, Obernosterer, F, Meyer, R, Huber, JB: On Prefilter Computation for Reduced-State Equalization. IEEE Trans. Wirel. Commun. 1(4), 793-800 (2002)

23. Eyuboglu, MV, Qureshi, SUH: Reduced-State Sequence Estimation with Set Partitioning and Decision Feedback. IEEE Trans. Commun. 36(1), 13-20 (1988)

24. Ziemann, O, Krauser, J, Zamzow, PE, Daum, W: POF Handbook: Optical Short Range Transmission Systems. 2nd Edition. Springer, Heidelberg (2008)

25. Proakis, J: Digital Communications. Fourth Edition. McGraw Hill, New York (2001)

26. Peng, L, Hélard, M, Haese, S: On Bit-loading for Discrete Multi-tone Transmission over Short Range POF Systems. IEEE/OSA J. Light. Technol. 31(24), 4155-4165 (2013)

27. Chatelain, B, Gagnon, F: Peak-to-average power ratio and inter symbol interference reduction by Nyquist pulse optimization. In: Proc. of IEEE 60th Vehicular Technology Conference. vol. 2, pp. 954-958. SPIE, Los Angeles, (2004)

\section{Submit your manuscript to a SpringerOpen ${ }^{\circ}$ journal and benefit from:}

- Convenient online submission

- Rigorous peer review

- Open access: articles freely available online

- High visibility within the field

- Retaining the copyright to your article

Submit your next manuscript at $\boldsymbol{\wedge}$ springeropen.com 